\title{
Internal transcribed spacer (ITS) sequence-based characterization of fungal isolates from multiple yogurt facilities-A case study
}

\author{
A. J. Buehler, R. L. Evanowski, M. Wiedmann, ${ }^{*}$ and N. H. Martin \\ Milk Quality Improvement Program, Department of Food Science, Cornell University, Ithaca, NY 14853
}

\begin{abstract}
Fungal spoilage remains a significant issue in dairy product quality, especially for cultured dairy products such as yogurt formulated without preservatives such as potassium sorbate. Fungal contamination can occur throughout the processing continuum, from the dairy farm environment to the finished product processing environment. As molecular characterization of fungal isolates is used more frequently, we obtained fungal isolates obtained in 2 yogurt processing facilities as part of routine fungal testing of raw materials (e.g., fruit preparations, added ingredients), in-process product samples, environmental samples (e.g., air plates, equipment surfaces such as valves, face plates, air nozzles), and finished product samples, to determine whether internal transcribed spacer (ITS) barcoding data would be helpful to support source tracking of fungal contamination issues. Internal transcribed spacer PCR amplification and sequencing allowed us to classify the 852 isolates from these 2 facilities into 200 unique ITS allelic types (AT), representing the phyla Ascomycota (743 isolates), Basidiomycota (97 isolates), and Mucoromycota (12 isolates). Thirty ITS AT were isolated from both facilities; 62 and 108 ITS AT were isolated from only facility A or only facility B, respectively. Nine ITS AT were each represented by more than 20 isolates; these AT comprised $53 \%$ of the 852 isolates. The considerable diversity of fungal isolates even within a single facility illustrates the challenge associated with controlling fungal contamination of dairy products. The ITS barcoding technique, however, did show promise for facilitating the source tracking of fungal contamination, particularly for ITS AT over-represented in a given facility. For example, we found evidence for equipment-specific reservoirs for 2 AT (14 and 219) in facility B. Our data suggest that despite its limited discriminatory power, ITS sequencing can provide initial information that can
\end{abstract}

Received September 1, 2018.

Accepted December 19, 2018

*Corresponding author: mw16@cornell.edu help trace fungal contamination along the processing continuum. However, development and implementation of discriminatory subtyping methods will be needed to further improve the ability to identify sources of fungal contamination in dairy facilities. Developing and implementing sampling plans that comprehensively capture yeast and mold diversity in a given processing facility remain a considerable challenge.

Key words: yogurt, yeast, mold, internal transcribed spacer (ITS) sequencing

\section{INTRODUCTION}

Fungal organisms are well documented to cause dairy product spoilage (Ledenbach and Marshall, 2009; Garnier et al., 2017). Global estimates on food loss suggest that 5 to $10 \%$ of the food supply is lost due to fungal spoilage (Pitt and Hocking, 2009). Dairy products, including cultured dairy products such as yogurt, without preservatives are particularly susceptible to fungal spoilage. Many fungal organisms are able to grow at refrigeration temperatures and at the low $\mathrm{pH}$ encountered in yogurt products (Fleet, 2011; Snyder et al., 2016); fungal isolates also frequently produce lipolytic and proteolytic enzymes that result in physical degradation and undesirable sensory characteristics in the product (Suriyarachchi and Fleet, 1981; Fleet and Mian, 1987; Ledenbach and Marshall, 2009). For example, a study in Australia characterized yeasts found in yogurts purchased from retail and found that the most frequently isolated species, Torulopsis candida and Kluyveromyces fragilis, were able to hydrolyze casein and produce bitter flavors (Suriyarachchi and Fleet, 1981). Fungal spoilage of yogurt can also lead to visible defects such as bloated containers and surface mycelial growth (Snyder et al., 2016). These fungal spoilage defects represent a significant financial loss when detected and product is destroyed and a business risk in the age of social media, when consumers can readily share pictures of dairy product quality defects (Newkirk et al., 2012). To reduce the risk of fungal spoilage of finished product, companies producing dairy products susceptible to mold growth often perform routine yeast 
and mold monitoring of their environment, raw materials, and finished product, with collection of substantial numbers of samples.

Fungal contamination can occur throughout the dairy processing continuum. Fungal organisms have been isolated from the dairy farm environment (Lavoie et al., 2012; Atanassova et al., 2016) and in the processing facility (Kure et al., 2003, 2008; Lund et al., 2003). Although fungal organisms have been reported in raw milk (Lavoie et al., 2012; Buehler et al., 2017), these organisms are not typically heat resistant and thus not a main source of fungal contamination in finished products. The processing facility environment, especially after pasteurization, represents a common source for fungal organisms that contaminate dairy products (Kure et al., 2003, 2004). In addition, ingredients used in yogurts (e.g., fruit preparations) may also be a source of fungal contaminants, typically yeasts (Suriyarachchi and Fleet, 1981). Although previous studies have tracked fungal organisms in cheese processing facilities (Kure et al., 2003; Lund et al., 2003; Mounier et al., 2006; Temelli et al., 2006), we are not aware of any published studies that used molecular methods to elucidate transmission routes and sources of fungal organisms in yogurt processing facilities. The internal transcribed spacer (ITS) region is the universal barcode for fungi and it allows for discriminatory DNA sequencing-based taxonomic characterization of fungal isolates (Mayoral et al., 2005; Schoch et al., 2012; Hibbett and Taylor, 2013). With increasing use and reduced cost of ITS barcoding approaches to identify fungal isolates, ITS barcoding data may increasingly become available to the dairy industry. Although species identification of fungal isolates (based on ITS sequence data) provides useful initial information for dairy processors that try to minimize product contamination with fungal spoilage organisms, the industry needs a better way to identify likely sources and transmission routes of fungal spoilage organisms. The ITS DNA sequencing technique is generally only used for species (or genus) identification and, in some cases, identifies multiple different ITS sequence types (ST) within a species; we are not aware of any publications that used ITS sequencing as a lowresolution tool to help identify likely fungal contamination sources in food processing facilities or other environments. We hypothesized that ITS sequencing may provide valuable and increasingly accessible data that could support source tracking and root cause analysis efforts for fungal contamination issues in the dairy industry. We therefore performed ITS DNA sequencing of fungal isolates obtained as part of company routine monitoring programs in 2 yogurt processing facilities.
Although we appreciate the potential shortcomings of this approach (e.g., lack of standardized set of sampling sites), it allowed us to obtain a large set of isolates and provided data on the potential use of barcoding data in conjunction with existing routine surveillance programs already implemented by industry.

\section{MATERIALS AND METHODS}

Isolates were obtained from raw material samples, finished product samples, in-process product samples, and environmental samples collected from 2 yogurt processing facilities, hereafter facilities A and B, in the United States. No data on the sample denominator (i.e., total number of samples collected by year) were available; although this represents a shortcoming, it is not unusual for industry to not have these data readily available. Samples were collected over 3 years (2015-2017) as part of routine monitoring in each facility; these programs typically represent convenient sampling plans with uneven sample sizes between facilities, seasons, and so on. Raw material samples included fruit preparations and other added ingredients; raw milk was not included as a raw material sample because dairy-relevant fungi are typically heat sensitive and thus raw milk would not represent a direct source of fungal contamination (Garnier et al., 2017). Finished product samples were collected as entire containers. In-process product samples were collected after pasteurization and included product samples from fermentation "maturation" tanks and from separators. Environmental samples represented (1) samples from equipment surfaces often suspected to be sources of fungal contamination (e.g., valves, face plates, air nozzles), collected using sponge-sticks (3M, St. Paul, MN) or swab-samplers (3M), and (2) air plate sampling on dichloran rose bengal chloramphenicol (DRBC; Becton, Dickinson and Co., Sparks, MD) agar using the sedimentation method (Dyer et al., 2004). For sponge and swab environmental samples, as well as raw material samples, isolates were obtained, using standard methods, on Petrifilm Yeast and Mold Count Plates (3M) and subsequently streaked for purity on DRBC agar or potato dextrose agar (Becton, Dickinson and Co.). For finished product samples, isolates were obtained using standard methods on DRBC agar or potato dextrose agar. The obtained fungal isolates were then characterized by DNA sequencing of the ITS region, using the ITS primers and thermocycling conditions described in Buehler et al. (2017). The ITS allelic types (AT) were assigned using EMBOSS Needle v.6.6.0.0 (Rice et al., 2000). A unique ITS AT was assigned for every ITS region sequence that differed by 
$\geq 1$ nucleotides from any previously obtained sequence described either in Buehler et al. (2017) or in this study; we appreciate that this approach includes a risk that sequencing artifacts or errors may result in different ITS AT and may be challenging for fungal isolates with intragenic heterogeneities involving the ITS region. The first isolate of each new ITS AT was designated as the reference strain for that AT; ITS AT designations are consistent with those reported by Buehler et al. (2017). Species identification was performed as described in Buehler et al. (2017), using the curated UNITE database (https://unite.ut.ee/analysis.php) to query ITS region sequences. If UNITE failed to provide genus and species identification with a $99 \%$ similarity threshold, the uncurated Mycobank database (www.mycobank.org) was used to query the sequences and provide genus and species identification where possible. Isolate characteristics and relevant sample information can be found at www.foodmicrobetracker.com using the "Search by Reference" function (http://www.foodmicrobetracker .com/search/SearchByReference.aspx; Vangay et al., 2013). Supplemental File S1 (https://doi.org/10.3168/ jds.2018-15636) provides a list of all isolates, including their taxonomic identification and AT. In addition, the DNA sequence data for all isolates are available as a FASTA file (Supplemental File S2; https://doi.org/10 .3168/jds.2018-15636) and in GenBank under accession numbers MK267356 to MK268207.

All statistical analyses were performed in R (v. 3.3.2; $\mathrm{R}$ Core Team, 2013). The frequency distributions of ITS AT between processing facilities and among all 4 sample types within processing facilities were compared using chi-squared tests of independence. As chi-squared tables included $>20 \%$ of cells with expected values $<5$, Monte Carlo simulations were used to estimate $P$ values. For pairwise chi-squared test comparisons of the distribution of ITS AT between individual sample types within a single processing facility (e.g., the distribution of ITS AT between in-process product samples and finished product samples in facility A), $P$-values were estimated using Monte Carlo simulations and were then adjusted using the Benjamini and Yekutieli (2001) method for multiple comparisons. To test for overrepresentation of ITS AT in facility A or B, pairwise Fisher exact tests were used to compare the frequency of a given ITS AT (for AT isolated $>10$ times) to the frequency of all other ITS AT.

\section{RESULTS AND DISCUSSION}

Overall, 852 isolates were characterized by ITS PCR amplification and sequencing; these isolates were classified into 200 unique ITS AT. The isolates were classified into 3 phyla, including Ascomycota (743 isolates), Basidiomycota (97 isolates), and Mucoromycota (12 isolates). The 303 isolates collected from facility A represented 92 ITS AT, and the 549 isolates collected from facility B represented 138 ITS AT. Thirty ITS AT were isolated from both facilities, whereas 62 and 108 AT were isolated from only either facility A or B, respectively. Nine ITS AT (AT 60, AT 145, AT 30, AT 101, AT 51, AT 102, AT 61, AT 219, and AT 59) were each represented by more than 20 isolates; these AT comprised $53 \%$ of the 852 isolates (data for all 48 ITS AT represented by $\geq 3$ isolates is shown in Table $1)$. The ITS AT represented by only one isolate $(\mathrm{n}=$ 129 ) comprised $15 \%$ of the 852 isolates. The remaining $32 \%$ of the 852 isolates were represented by ITS AT containing $\geq 2$ and $\leq 20$ isolates. A chi-squared test of independence revealed a significant difference in the distribution of ITS AT between facilities A and B ( $P$ $<0.05)$. Moreover, pairwise Fisher exact tests [comparing the frequency of a given ITS AT (for AT that occurred $>10$ times) to the frequency of all other ITS AT] showed that ITS AT 30, AT 60, AT 101, AT 155, and AT 209 were over-represented in facility A, and ITS AT 14, AT 61, AT 145, and AT 219 were over-represented in facility B; hence, results for facilities A and B are discussed separately below.

In facility A, the most common ITS AT were identified as Torulaspora delbrueckii (AT 60, 58 isolates), T. delbrueckii (AT 101, 36 isolates), and Candida parapsilosis (AT 30, 29 isolates). Interestingly, the 3 most common ITS AT as well as the 5 ITS AT over-represented in facility A were all classified as yeasts. Molds were, however, represented among the remainder of isolates from facility A and represented 42/303 isolates from this facility. This observed over-representation of yeast compared with mold contaminants could suggest a focus on implementing yeast control strategies throughout this facility, whereas mold control strategies could be focused locally where mold contamination risk is elevated, such as in the filling and packaging area (Garnier et al., 2017) or areas where mold contaminants are found. In facility A, only 1 mold ITS AT from environmental sources (ITS AT 38) was obtained 3 times (the other 36 environmental mold isolates from facility A were obtained $\leq 2$ times); this low repeated isolation of mold ITS AT further complicates the use of these data for source tracking.

Among the 4 sample types in facility A (raw material, in-process product, environmental, and finished product samples), the majority of isolates (270/303 isolates) were collected from environmental samples or 


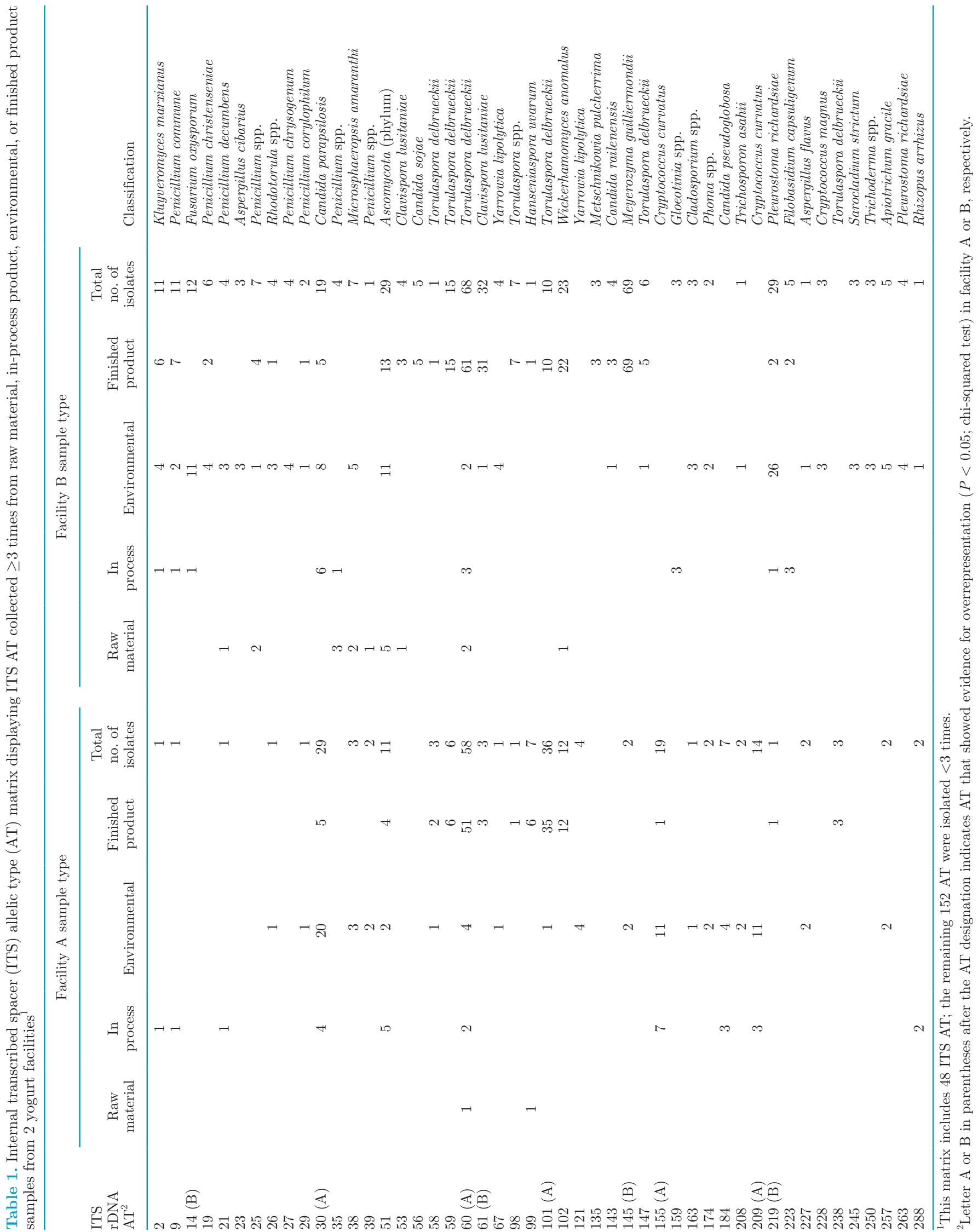


finished product samples. The overall distribution of ITS AT differed among all sample types $(P<0.05$, chi-squared test). Pairwise chi-squared tests were thus used to test for the association between individual sample types (in-process product, environmental, and finished product samples; raw material samples were not included because only 3 isolates were collected from these samples). Although the distribution of ITS AT between environmental samples and in-process product samples was not significantly different $(P \geq 0.05)$, suggesting the possibility of an environmental transmission route for in-process product contamination (Table 2), the distribution of ITS AT was significantly different $(P<0.05)$ between in-process product samples and finished product samples, as well as between environmental samples and finished product samples (Table 2 ). The null hypothesis (i.e., no significant difference in distribution of ITS AT between environmental samples and finished product samples) would provide some support that yeast and mold in environmental and product represent the same populations. Hence, rejection of the null hypothesis may indicate that sources other than the environmental sites sampled could contribute to product contamination or that some sites sampled contribute more to product contamination than others; these findings may also reflect incomplete sampling of the environment, which is likely to occur in routine sampling programs in processing facilities and contributes to the challenge of tracking fungal spoilage organisms along the processing continuum. Importantly, the limited routine sampling of raw materials in facility $\mathrm{A}$ also affected our ability to identify potential sources of yeast, which have been suspected to contaminate fruit preparations used in yogurt manufacturing (Suriyarachchi and Fleet, 1981). However, sterile collection of fruit preparation samples from large sealed fruit containers is a challenge, and samples may not reflect the microbiological conditions of all the fruit in the container, which potentially contributes to the low number of raw material samples collected.

The ITS AT data generated for facility A also illustrated the potential value of these data for source tracking, which we initially assessed by focusing on ITS AT over-represented in a given facility as these ITS AT are less likely to simply represent common subtypes. Among the 5 ITS AT over-represented in facility A, isolates characterized as ITS AT 101 were obtained over 6 mo and predominantly identified from a product with a specific fruit preparation ("fruit prep A"). Overall, 30 out of the 35 ITS AT 101 finished product isolates were from products with "fruit prep A," suggesting either the raw material or specific line or equipment used for this yogurt was a source. Interestingly, a second ITS AT (AT 60) that was over-represented in facility A (and isolated from there over $2 \mathrm{yr}$ ) was also the second most common AT found in facility B. Although repeat isolation in a given facility of more widely distributed ITS AT such as this may provide some potentially valuable hypotheses on contamination sources, subtyping methods with greater resolution power (e.g., sequencing protein-coding genes, fragment analysis techniques, microsatellites, random amplified polymorphic DNA, whole-genome sequencing; Almeida and Araujo, 2013; Saghrouni et al., 2013) may be needed to facilitate reliable source tracking, particularly for common and widely distributed ITS AT.

In facility $\mathrm{B}$, the most common ITS AT were identified as Meyerozyma guilliermondii (AT 145, 69 isolates), T. delbrueckii (AT 60, 68 isolates), and Clavispora lusitaniae (AT 61, 32 isolates). Interestingly, 2 Byssochlamys isolates (AT 36) were collected from raw material and in-process samples from facility B (Supplemental File S1; https://doi.org/10.3168/jds.2018 -15636). Byssochlamys is one of the most heat-resistant food-associated fungi that is known (Houbraken et al., 2008), with D-values of approximately $10 \mathrm{~min}$ at $88^{\circ} \mathrm{C}$

Table 2. Association between sample type and internal transcribed spacer (ITS) allelic type (AT) within each yogurt processing facility; numbers represent chi-squared test statistic $(P \text {-values in parentheses })^{1,2}$

\begin{tabular}{lcccc}
\hline & & \multicolumn{2}{c}{ Facility A } \\
\cline { 2 - 5 } Facility B & $\begin{array}{c}\text { Raw material } \\
(\mathrm{n}=3)\end{array}$ & $\begin{array}{c}\text { In process } \\
(\mathrm{n}=30)\end{array}$ & $\begin{array}{c}\text { Environmental } \\
(\mathrm{n}=125)\end{array}$ & $\begin{array}{c}\text { Finished product } \\
(\mathrm{n}=145)\end{array}$ \\
\hline Raw material $(\mathrm{n}=31)$ & & $\mathrm{ND}^{3}$ & $\mathrm{ND}$ & $\mathrm{ND}$ \\
In process $(\mathrm{n}=25)$ & $\begin{array}{c}14.1(<0.05) \\
\text { Environmental }(\mathrm{n}=187)\end{array}$ & $131(\geq 0.05)$ & $62.5(\geq 0.05)$ & $124(<0.05)$ \\
Finished product $(\mathrm{n}=306)$ & $224(<0.05)$ & $199(<0.05)$ & $397(<0.05)$ & $221(<0.05)$ \\
\hline
\end{tabular}

${ }^{1}$ As chi-squared tables included $>20 \%$ of cells with expected values $<5$, Monte Carlo simulations were used to estimate $P$-values. The $P$-values were then adjusted for multiple comparisons using the Benjamini and Yekutieli (2001) method. Numbers above the diagonal represent comparisons within facility A, and numbers below the diagonal represent comparisons within facility B.

${ }^{2} \mathrm{n}$ represents the total number of isolates collected from the specific sample type in a given facility.

${ }^{3}$ Analyses were not performed due to small sample size. 
(King et al., 1969). The presence of these heat-resistant fungi in raw materials may represent a significant challenge for manufacturers. The 3 most common ITS AT, as well as 3 of 4 ITS AT over-represented in facility $\mathrm{B}$, represented yeasts. One over-represented ITS AT in facility B represented a mold (ITS AT 219; Pleurostoma richardsiae); ITS AT 219 was only found once among the isolates from facility A. In facility B, this AT was isolated 26 times from environmental samples but only once and twice from in-process product and finished product samples, respectively, suggesting its potential as an environmental reservoir or source in facility B (also supported by the fact that 8 of 26 environmental ITS AT 219 isolates were obtained from samples associated with a specific piece of equipment). These findings further illustrate the value of ITS barcoding for source tracking. The 3 most common ITS AT in facility $\mathrm{B}$ represented 169 isolates; interestingly, 161 of these isolates were collected from finished product samples. Infrequent isolation of yeasts from other sample types could be a consequence of sampling strategies and sample site selection, which may have biased results toward the recovery of molds. As yeast contamination is typically associated with added ingredients or improper cleaning and sanitation (Garnier et al., 2017), intensive sampling of raw materials (or sites affected by raw materials) or internal equipment areas that are challenging to clean and sanitize may facilitate yeast isolation, whereas collection of air samples may bias sampling toward isolation of molds. Hence, the nature of routine environmental sampling plans needs to be carefully considered when assessing yeast and mold contamination patterns and subtype data. Although we are not aware of specific guidance for developing environmental sampling plans for yeasts and molds, guidance documents on sampling plans for environmentally transmitted foodborne pathogens (e.g., Listeria; Innovation Center for US Dairy, 2017; FDA, 2017) may represent a good starting point for the design of improved yeast and mold monitoring programs.

Among the 4 sample types, the majority of isolates $(493 / 549)$ in facility B were collected from finished products and environmental samples. The distribution of ITS AT differed among all sample types (raw material, in-process product, environmental, finished product) collected from facility B $(P<0.05$, chi-squared test). Pairwise chi-squared tests for the association between ITS AT and individual sample types showed that ITS AT distributions for (1) raw materials, (2) in-process product, and (3) environmental samples were significantly different $(P<0.05)$ from the distribution of ITS AT from finished product samples (Table 2), which, as discussed for facility A, may reflect incomplete sam- pling of these different sample types, but could also be explained by multiple sources contributing to finished product contamination.

Similar to facility A, a qualitative assessment of ITS AT over-represented in facility B illustrates the potential value of these data for source tracking. In addition to suggesting a possible specific source of Pleurostoma richardsiae (ITS AT 219) in facility B (as discussed above), our data suggested that ITS AT 14 (isolated only in facility B) may have a specific reservoir, because 8 of 12 isolates with this AT were isolated from air nozzles. In addition, for ITS AT 145, 31/69 finished product isolates were produced on a single line, which could indicate a line-specific issue or possibly, but less likely, an issue associated with a specific type of product predominantly manufactured on this line. Finally, ITS AT 61 was represented by 32 isolates from facility B (compared with only 3 isolates from facility A); 22 isolates were obtained from 2 different lines (10 and 12 isolates), with 2 yogurt types (with fruit preps B and C) produced on both lines. These findings may indicate a source for ITS 61 that is common to both lines; this type of information can be valuable for guiding further in-plant root cause analysis efforts. Overall, the examples provided illustrate how ITS barcoding data can be valuable to suggest specific directions for root cause analyses, even if the data do not necessarily point to a specific source. Our findings are consistent with a previous study (Kure et al., 2003) that showed some fungal strains (as identified by amplified fragment length polymorphism) to be associated with specific cheese processing facilities with repeat isolation over $>1 \mathrm{yr}$, which could suggest persistence of a given strain in a processing facility. Similar to our study, Kure et al. (2003) found that some fungal strains were specifically associated with a certain location in a facility.

Overall, our data revealed that routine monitoring programs for fungal contamination in dairy processing facilities capture considerable fungal diversity, with 200 different subtypes (ITS AT) found across 2 facilities. Moreover, we showed that ITS sequencing provides initial information that can help identify possible fungal contamination sources or at least help develop specific hypotheses for root cause analysis efforts targeting fungal contamination issues, demonstrating the value of these methods for industry. However, our data also indicate the presence of common and widely distributed ITS subtypes, consistent with the fact that ITS barcoding is targeted to allow species identification, not subtyping. This enforces the need for discriminatory subtyping methods to definitively identify contamination sources. This is also supported by a previous study that found the same ITS AT in different dairy products 
(e.g., cheese and yogurt; Buehler et al., 2017). The value of ITS-based barcoding may be affected not only by the limited discriminatory power of this method, but also by the sensitivity of the approach described here to sequencing artifacts or errors and intragenic heterogeneities involving the ITS region, which may occur in some fungal species.

\section{CONCLUSIONS}

As fungal contamination of yogurt as well as other dairy products will likely continue to represent a challenge, particularly considering that multiple routes for fungal contamination exist along the processing continuum (e.g., added ingredients, environmental contamination), industry will need to continue to implement improved control strategies for these spoilage organisms, using a holistic systems approach. Our data suggest that robust approaches to identify and control fungal spoilage should represent a combination of (1) raw material sampling; (2) well-designed environmental sampling programs targeting likely sources of both yeast and mold contamination, with collection of appropriate sampling site metadata; and (3) use of characterization and subtyping methods, including methods with improved resolution over ITS barcoding when needed. Although fungal spoilage organisms are not of public health significance, investment into the use of characterization methods for fungal isolates facilitates elucidation of sources during root cause analysis and thus has considerable value. This approach will typically show a return on investment when considering the full costs of destroying and managing spoiled or contaminated product. Sanitation and equipment preventive maintenance are key programs in the manufacturing of products without preservatives, and the use of molecular methods, such as ITS barcoding, will facilitate proper implementation and execution of these programs. In addition, early detection and proper disposition of contaminated product not only reduce the risk of spoiled product reaching consumers, but can also provide isolates for characterization and subtyping to facilitate root cause analyses.

\section{ACKNOWLEDGMENTS}

The authors thank the staff of the Milk Quality Improvement Program (Cornell University, Ithaca, NY), specifically Sherry Roof, for assistance with this study. This project was partially supported by the New York State Dairy Promotion Advisory Board.

\section{REFERENCES}

Almeida, L. A., and R. Araujo. 2013. Highlights on molecular identification of closely related species. Infect. Genet. Evol. 13:67-75.

Atanassova, M. R., C. Fernández-Otero, P. Rodríguez-Alonso, I. Fernández-No, J. Garabal, and J. Centeno. 2016. Characterization of yeasts isolated from artisanal short-ripened cows' cheeses produced in Galicia (NW Spain). Food Microbiol. 53:172-181.

Benjamini, Y., and D. Yekutieli. 2001. The control of the false discovery rate in multiple testing under dependency. Ann. Stat. 29:1165-1188.

Buehler, A. J., R. Evanowski, N. Martin, K. Boor, and M. Wiedmann. 2017. Internal transcribed spacer (ITS) sequencing reveals considerable fungal diversity in dairy products. J. Dairy Sci. 100:88148825 .

Dyer, R., J. Frank, B. Johnson, and P. Hickey. 2004. Microbiological tests for equipment, containers, water, and air. Pages 325-340 in Standard Methods for Examination of Dairy Products. Vol. 17. 16th ed. American Public Health Association, Washington, DC.

FDA (Food and Drug Administration). 2017. Chapter 13: Environmental monitoring to verify control of Listeria spp. or L. monocytogenes. In Draft Guidance for Industry: Control of Listeria monocytogenes in Ready-To-Eat Foods. Accessed Nov. 14, 2018. https:// www.fda.gov/RegulatoryInformation/Guidances/ucm073110.htm;

Fleet, G. H. 2011. Chapter 5: Yeast spoilage of foods and beverages. Pages 53-63 in The Yeasts: A Taxonomic Study. 5th ed. C. Kurtzman, J. W. Fell, and T. Boekhout, ed. Elsevier, London, UK.

Fleet, G. H., and M. A. Mian. 1987. The occurrence and growth of yeasts in dairy products. Int. J. Food Microbiol. 4:145-155.

Garnier, L., F. Valence, and J. Mounier. 2017. Diversity and control of spoilage fungi in dairy products: An update. Microorganisms 5:42.

Hibbett, D. S., and J. W. Taylor. 2013. Fungal systematics: Is a new age of enlightenment at hand? Nat. Rev. Microbiol. 11:129.

Houbraken, J., J. Varga, E. Rico-Munoz, S. Johnson, and R. A. Samson. 2008. Sexual reproduction as the cause of heat resistance in the food spoilage fungus Byssochlamys spectabilis (anamorph Paecilomyces variotii). Appl. Environ. Microbiol. 74:1613-1619.

Innovation Center for US Dairy. 2017. Control of Listeria monocytogenes: Guidance for the U.S. dairy industry https://www.usdairy .com/ /media/usd/public/control-of-listeria-monocytogenes -guidance-for-the-us-dairy-industry.pdf? accessed 11/14/2018.

King, A. D., H. D. Michener, and K. A. Ito. 1969. Control of Byssochlamys and related heat-resistant fungi in grape products. Appl. Microbiol. 18:166-173.

Kure, C. F., E. Borch, I. Karlsson, J. P. Homleid, and S. Langsrud. 2008. Use of the selective agar medium CREAD for monitoring the level of airborne spoilage moulds in cheese production. Int. J. Food Microbiol. 122:29-34.

Kure, C. F., I. Skaar, and J. Brendehaug. 2004. Mould contamination in production of semi-hard cheese. Int. J. Food Microbiol. 93:41-49.

Kure, C. F., I. Skaar, A. Holst-Jensen, and E. Abeln. 2003. The use of AFLP to relate cheese-contaminating Penicillium strains to specific points in the production plants. Int. J. Food Microbiol. 83:195-204.

Lavoie, K., M. Touchette, D. St-Gelais, and S. Labrie. 2012. Characterization of the fungal microflora in raw milk and specialty cheeses of the province of Quebec. Dairy Sci. Technol. 92:455-468.

Ledenbach, L. H., and R. T. Marshall. 2009. Microbiological spoilage of dairy products. Pages 41-67 in Compendium of the Microbiological Spoilage of Foods and Beverages. Springer, New York, NY.

Lund, F., A. B. Nielsen, and P. Skouboe. 2003. Distribution of Penicillium commune isolates in cheese dairies mapped using secondary metabolite profiles, morphotypes, RAPD and AFLP fingerprinting. Food Microbiol. 20:725-734.

Mayoral, M. B., R. Martín, A. Sanz, P. E. Hernández, I. González, and T. García. 2005. Detection of Kluyveromyces marxianus and other spoilage yeasts in yoghurt using a PCR-culture technique. Int. J. Food Microbiol. 105:27-34. 
Mounier, J., S. Goerges, R. Gelsomino, M. Vancanneyt, K. Vandemeulebroecke, B. Hoste, N. Brennan, S. Scherer, J. Swings, and G. Fitzgerald. 2006. Sources of the adventitious microflora of a smear-ripened cheese. J. Appl. Microbiol. 101:668-681.

Newkirk, R. W., J. B. Bender, and C. W. Hedberg. 2012. The potential capability of social media as a component of food safety and food terrorism surveillance systems. Foodborne Pathog. Dis. 9:120-124.

Pitt, J. I., and A. D. Hocking. 2009. Fungi and Food Spoilage. 3rd ed. Springer, New York, NY.

R Core Team. 2013. R: A language and environment for statistical computing. R Foundation for Statistical Computing, Vienna, Austria.

Rice, P., I. Longden, and A. Bleasby. 2000. EMBOSS: The European molecular biology open software suite. Trends Genet. 16:276-277.

Saghrouni, F., J. Ben Abdeljelil, J. Boukadida, and M. Ben Said. 2013. Molecular methods for strain typing of Candida albicans: A review. J. Appl. Microbiol. 114:1559-1574.
Schoch, C. L., K. A. Seifert, S. Huhndorf, V. Robert, J. L. Spouge, C. A. Levesque, W. Chen, and F. B. Consortium. 2012. Nuclear ribosomal internal transcribed spacer (ITS) region as a universal DNA barcode marker for fungi. Proc. Natl. Acad. Sci. USA 109:6241-6246.

Snyder, A. B., J. J. Churey, and R. W. Worobo. 2016. Characterization and control of Mucor circinelloides spoilage in yogurt. Int. J. Food Microbiol. 228:14-21.

Suriyarachchi, V. R., and G. H. Fleet. 1981. Occurrence and growth of yeasts in yogurts. Appl. Environ. Microbiol. 42:574-579.

Temelli, S., S. Anar, C. Sen, and P. Akyuva. 2006. Determination of microbiological contamination sources during Turkish white cheese production. Food Control 17:856-861.

Vangay, P., E. B. Fugett, Q. Sun, and M. Wiedmann. 2013. Food microbe tracker: a web-based tool for storage and comparison of food-associated microbes. J. Food Prot. 76:283-294. 